\title{
High MRSA Carriage Rate among Nursing Microbiology Students
}

\author{
Alan Lee Gillen*, Whitney 0. Daycock, Andrew Serafin \\ Department of Biology \& Chemistry, Liberty University, Lynchburg, VA, USA \\ Email: ${ }^{*}$ algillen@liberty.edu \\ Received 15 July 2014; revised 10 August 2014; accepted 9 September 2014 \\ Copyright (C) 2014 by authors and Scientific Research Publishing Inc. \\ This work is licensed under the Creative Commons Attribution International License (CC BY). \\ http://creativecommons.org/licenses/by/4.0/

(c) (i) Open Access

\begin{abstract}
Staphylococcus aureus is a common cause of disease, particularly in colonized persons. Although methicillin-resistant $S$. aureus (MRSA) infections have become increasingly reported, populationbased studies of students preparing for the health professions having $S$. aureus and MRSA colonization are lacking. We have found that students in microbiology classes having more contact with individuals in a healthcare setting are more likely to carry MRSA in their nares and axilla. The classes who had the highest rate of MRSA carriage during the school year, 2013-2014, were those with nursing students, who had a greater exposure to clinical settings and nursing homes. The class which had the highest rate of $S$. aureus carriage, had nearly $50 \%$ of the infected students had been involved a clinical setting. Since the majority of the students in the nursing and biology courses are looking to pursue a career in medicine, this sampling was very beneficial to inform them and others if they were a carrier of $S$. aureus and MRSA and the preventative measures to reduce the risk of infection.
\end{abstract}

\section{Keywords}

Staphylococcus aureus, MRSA, Staphylococcus Carriage, Clinical Student Risks

\section{Introduction}

Serious methicillin resistant Staphylococcus aureus (MRSA) infections are an increasing global concern according to the CDC in 2013 [1]. The CDC reports that two million people have become infected with antibiotic resistant bacteria with 80,461 severe MRSA infections and 11,285 deaths per year in 2013 [1]-[3]. Hosts can carry Staphylococcus aureus and MRSA asymptomatically with these bacteria are commonly found in their nares and axilla [4] [5]. According to numerous studies, about 33\% of people are Staphylococcus carriers [6]. Given the

${ }^{*}$ Corresponding author. 
growing concern of antibiotic resistance, we determined the local prevalence of staphylococci and MRSA colonizations in students preparing for health professions. The study was conducted during the school year of 20132014, thus representing a very current trend of students training for the health professions. Although MRSA infections are increasingly reported in hospitals and communities, there have been very few studies among healthcare students under training. The last study published on a similar population was in 2004 (10 years ago) in France [7]. Our main contribution was answering the question: Is nasal carriage of MRSA more prevalent among students who have clinical exposures? Secondary contributions of our investigation include multiple body sites carriage and the prevalence of multiple MRSA biotypes in light of recent reports of multiple strain carriage [4] [5].

\section{Methods}

Students were sampled at Liberty University in various microbiology classes to compare carriage of Staphylococcus $s p$. between those exposed to time in a clinical setting (one week or more) and those individuals not exposed to a clinical environment. In order to determine the prevalence of $S$. aureus carriers among individuals, several biology classes from Liberty University were examined. The bacterial isolates and culture swabs in this work were selected from those collected in our study of S. aureus and MRSA colonization in college students scheduled for microbiology class in their normal MRSA detection lab. Nasal swabs were obtained during lab time where students had already been instructed in sampling technique, plating and growing bacteria. This sample collection protocol and survey study was approved by the Liberty University institutional review board (IRB). It insured anonymity of all participants with an 11-question biomedical survey, results and procedures.

The student surveys were divided by academic class and section; the specific classes tested were Biology 203, 303, and 305 at Liberty University. The Biology 203 students consisted mainly of nursing or health-promotion majors, in which the majority had exposure in a clinical setting. The Biology 303 and 305 students consisted mainly of biology majors, the majority of which have little exposure in clinical settings. The first step was to swab the student's left and right nares as well as left and right axilla, using a separate, sterile cotton swab for each sample. The students streaked the contents from each swab onto a designated quadrant on a Petri plate with either Mannitol salt agar (MSA) or CHROMagar ${ }^{\mathrm{TM}}$ Staph aureus, both of which specifically test for Staphylococcus growth. MSA contains a high concentration (7.5\%) of salt, making it selective for members of the family Micrococcaceae and Staphylococcus, since this level of NaCl is inhibitory to most other bacteria. CHROMagarM Staph aureus was used as a control for the experiment, to ensure the validity of $S$. aureus growth. The plates were incubated at $37^{\circ} \mathrm{C}$ for 48 hours. In addition to obtaining a culture sample, each individual was asked to complete an anonymous survey with a correlated number to place on his or her plates. The survey included questions that could lead to potential reasons for carriage.

Once the incubation was complete, the plates were examined for any bacterial growth. If yellow cultures were present on the MSA, this marked a positive for S. aureus growth. The Staphylococcus culture was then transferred onto a MSA plate containing oxacillin to test for the presence of MRSA. Oxacillin, which is in the same class of drugs as methicillin, was chosen as the agent of choice for testing due to its chemical stability. For those MRSA positive, we also followed Kirby-Bauer procedure for antibiotic sensitivity using Mueller-Hinton plates.

Isolates were classified as susceptible or resistant to the antibiotics based on standardized tables given for the Kirby-Bauer test and those for S. aureus in the ASM antibiogram manual [8]. For antibiogram analysis, S. aureus standard strains used as control were obtained from Carolina Biological Supply Co. The control strain was Staphylococcus aureus subsp. aureus Rosenbach (ATCC ${ }^{\circledR} 43300^{\mathrm{TM}}$ ) Strain Designation: F-182.

CHROMagar ${ }^{\mathrm{TM}}$ Staph aureus and MRSA Select were also agar types used for determining questionable MSA results and clarifying Staph and MRSA colony variations. Both agar types are chromogenic and display various color intensities that predict variation among S. aureus biotypes (or strains). We also tested for Coagulase Negative Staphylococcus (CoNS) bacteria in all sections as a control. Coagulase-Negative staphylococci (CoNS) are a broad group of species that commensally inhabit the human skin, mucous membranes (S. hominis, S. epidermidis), and other body surfaces (S. saprophyticus). Although they are less virulent than the coagulase-positive $S$. aureus and almost never pathogenic in healthy individuals, their universal presence demonstrates that each section sampled correctly and media supported growth. These bacteria represent normal microbiota and are considered nonpathogens for these healthy students.

\section{Statistical Analyses}

Standard descriptive statistics were made for prevalence of staphylococci and MRSA abundance with each class 
examined. Numbers of positive and negative individuals in different groups were tabulated in $2 \times 2$ contingency tables and compared with a $\chi^{2}$ test. $\mathrm{p}$ values as given in the text refer to the probabilities that the differences were due to sampling error.

\section{Results}

\subsection{Prevalence of Staph and MRSA Carriage}

In an initial 2013 study, 45\% of the individuals in nursing microbiology classes $(\mathrm{N}=42)$ were $S$. aureus carriers and $19 \%$ had MRSA. In general microbiology classes $(\mathrm{N}=42)$, six $(14 \%)$ were $S$. aureus carriers and only one (2\%) was a MRSA carrier. The differences in carrier rates between nursing students and general microbiology students are statistically significant $\left(\chi^{2}, \mathrm{p}=0.014\right.$ for $S$. aureus carrier rate and $\chi^{2}, \mathrm{p}=0.0019$ for MRSA carrier rate).

A subsequent 2014 study (Figure 1, Figure 2) was conducted to collect a more thorough data ( $\mathrm{N}=164$ students). In this study, students in various classes were swabbed in both nostrils and axilla. We found that students involved in a (one or more) clinical setting had a higher percentage of carrying $S$. aureus and MRSA. In the nursing microbiology (BIOL 203) classes, nearly 25\% of the students tested were MRSA carriers, and of those, $60 \%$ were active in a clinical or nursing home environment. There were four $(16.7 \%)$ students in an advanced microbiology class (BIOL 305) that carried MRSA, with three of these involved a clinical setting. The general microbiology (BIOL 203) class had a similar MRSA carriage rate (17.5\%), with 43\% of those being involved in clinical work. There is no statistical significance in the differences of carrier rates between the class "type" alone. However, when we examined clinical vs. non-clinical status, we found a significant difference in MRSA carriage $\left(\chi^{2}, \mathrm{p}=0.068\right)$. Students involved with clinical care increased their risk of picking up and carrying MRSA.

Our overall results (of 32.3\%) are consistent with other national studies (7) for S. aureus carriage. However, the MRSA carriage rates among nursing microbiology students were much higher (24\%) than the national average $(1 \%-3 \%)$. The nursing students who are involved in clinical settings as part of their training along with students who work in a clinical settings had the highest rate of MRSA carriage. Most MRSA carriers (60\%) for all microbiology classes were involved in a clinical setting as well. The heaviest MRSA colonization (by colony count) came from four students that worked in a nursing home.

\subsection{Anatomy of MRSA Carriage}

Staphylococcus aureus can be carried and grown in many ecological niches in the human body. The nose, axilla, and perineum are among the most common anatomical sites. Typically, S. aureus is sampled only in the anterior nares because it is the most common carriage place. However, we have found about $2 \%$ of individuals that had it colonizing only the axilla and not the nose. Also, we found that about $2 \%$ had it in only one nostril and not both. We felt it important to sample four places (right and left anterior nares; right and anterior axilla). By sampling 4 different places on the body instead of just one or two, the incidence of MRSA for those who were non-clinical was shown to be much higher during spring, 2014 than results gathered in fall, 2013. It was still shown that working in a clinical setting causes a higher incidence of MRSA. Of the people tested in the BIOL 305 group, 4 out of 5 students with abnormal nasal anatomy tested positive for MRSA while none of 11 students with normal anatomy had MRSA $\left(\chi^{2}, \mathrm{p}=0.00061\right)$, indicating that abnormal nasal anatomy increased the risk of having MRSA.

Because Staphylococcus aureus is becoming more and more difficult to control with antibiotics, it is imperative that habits and characteristics that make someone more susceptible to carriage be identified. With this information, safety measures and education can begin to decrease the incidence for MRSA carriers that put people at risk for infection. Our study compared the accuracy of sampling volunteers in only one or two places (fall, 2013) compared to four different places (spring, 2014). As shown in the charts below, a much higher incidence of MRSA was detected in the nonclinical setting group when all four places were tested. This increased the accuracy for detection and led to more informed conclusions when comparing incidence to the risk factors asked about in the surveys.

Although most sections sampled showed inconclusive data for abnormal nasal anatomy, one section accurately described their internal nasal anatomy and was able to show a link between this risk factor and an increase in MRSA carriage. Our results showed that $80 \%$ of people with abnormal nasal anatomy were carriers of 


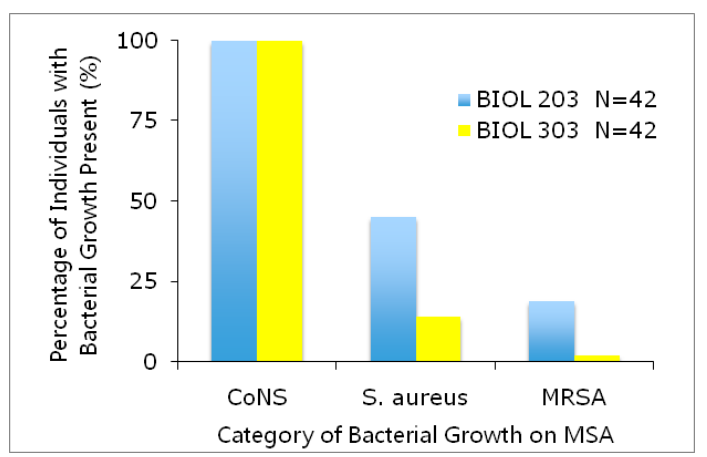

Figure 1. The fall, 2013 percentage of staphylococcal carriage between BIOL 203 and 303 students. CoNS = Coagulase Negative Staphylococcus bacteria.

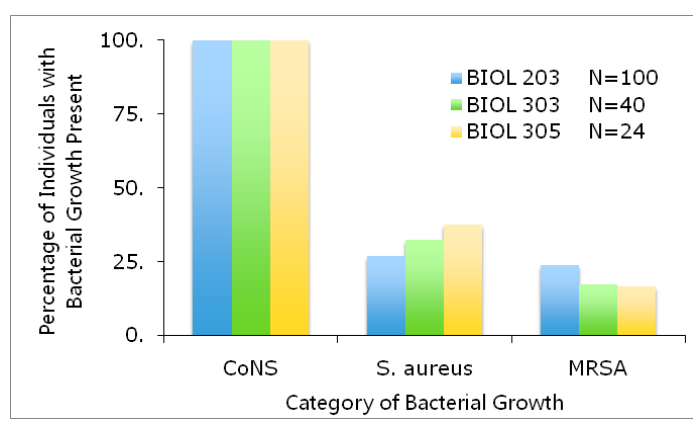

Figure 2. The spring, 2014 percentage of staphylococcal carriage between BIOL 203, 303, and 305 students. CoNS = Coagulase Negative Staphylococcus bacteria.

MRSA, while those with normal nasal anatomy did not test positive for MRSA. These findings agreed with other studies that have been done showing that having a deviated septum or enlarged turbinates can increase the likelihood of colonization by MRSA after exposure [9]. Future studies of MRSA will continue to sample multiple body sites and evaluation of nasal anatomy will also be evaluated.

\subsection{Diversity of Staph/MRSA "Types"}

MRSA Types: Following isolation streaks and growth in Trypticase Soy Broth (TSB), four MRSA strains were grown on Mueller-Hinton, MSA with oxacillin, and MRSA Select agars. MRSA biotypes or strains were determined by the methods given in the book, Antibiogram [8]. The difference in MRSA strains was seen directly by the zone sizes produced. The strain data was recorded for both resistance and inhibition sizes as seen in Table 1 .

Upon examination of the four MRSA strains (two from each of two people) were found to vary in their antibiotic sensitivity and resistance. One specific strain showed resistance to three different antibiotics. Two unique strains that were isolated from separate nares of the same person were sensitive to penicillin differently. The antibiogram patterns were distinct indicating various biotypes or strains [8]. Student "A" strains varied in sensitivity to vancomycin and two strains from student "B" varied in sensitivity to penicillin, erythromycin, and sulfa drugs.

\section{Discussion}

In all, we found 16 distinct bacterial isolates based upon antibiograms and other tests (data not shown). Although these isolates are distinct; they appear to be variants of local hospital acquired MRSA strains [10]. Among those who had MRSA, we found four individuals who had different bacterial isolates in the right and left anterior nares. In these students, $S$. aureus isolates were unique to each nostril. Two unique bacteria isolates were acquired from their separate nares and had distinct antibiotic sensitivity. In the four nursing students with distinct bacteria 
Table 1. Sample for antibiogram comparison zones of inhibition (mm) for antibiotic disc sensitivity tests on multiple MRSA isolates of same person.

\begin{tabular}{ccccc}
\hline Antibiotic/Drug Dosage & Student A Left Nare & Student A Right Nare & Student B Left Nare & Student B Right Nare \\
\hline DoxycyclineD-30 & 47 & 40 & 50 & 49 \\
Streptomycin S-10 & 47 & 18 & 46 & 41 \\
Vancomycin VA-30 & 38 & 7 & 39 & 32 \\
Erythromycin E-15 & 50 & 48 & 47 & 6 \\
Penicillin P-10 & 45 & 45 & 45 & 6 \\
Sulfa Drug SXT-25 & 43 & 40 & 41 & 6 \\
\hline
\end{tabular}

Note: Size of Disc = 6 mm; Dosage Unit is mcg; Average Zone Sizes with Multiple Replicates; Discs Obtained from Troy Biological Supply Co.

isolates growing on each side of the nose, it appears they have acquired different bacteria types from different sources. Each student had involvement with nursing homes. In the one specific case, the individual's nose had various piercings and had recently worked in a nursing home. In the other three cases, based upon interviews, it appears they acquired MRSA isolates from different patients. Over the past few years, the prevalence of MRSArelated hospital and nursing home infections has increased. Recently, a nursing home in California reported a MRSA prevalence of 26\% [11] [12].

One particular student, who had the heaviest bacteria colonization, shadowed an occupational therapist for a few weeks at a nursing home. In addition, we hypothesize that the individual got a pierced nose six months prior to MRSA testing. The strain in the left nose appeared like other MRSA isolates from students who had been in nursing homes, but the other isolate looked unique. We speculate the student may have picked up one MRSA isolate from her nursing home work and one from the facility that conducted ring piercings. Our data indicates that students who have worked in nursing homes and other clinical settings have developed a MRSA carriage prevalence of $16 \%$ - 25\%. We believe our findings have clinical implications, because in each case, one isolate from one nostril was mupirocin sensitive and the other mupirocin resistant. Given the common treatment of topical mupirocin, only partial elimination of MRSA would occur.

Staphylococcus aureus is an important pathogen responsible for a range of clinical entities from asymptomatic colonization to invasive and life-threatening infections. Carriage of $S$. aureus has been associated with subsequent infections, especially surgical site infections and a variety of other clinical conditions. Studies have revealed that among individuals with $S$. aureus infections, the infecting strains were indeed genetically identical or related to the carriage strains.

Only four studies have reported the carriage of multiple subtypes (or strains) of methicillin-resistant S. aureus (MRSA) swabbed from adult patients during their stay in hospitals [5]. It was uncertain whether these episodes were polyclonal as a result of multiple clones simultaneously colonizing patients, or acquisition of different MRSA strains over time. Several researchers have studied MRSA carriage at various body sites and found that multiple subtypes can be carried simultaneously at different sites by a single patient. However, the frequency of concurrent colonization with multiple genotypes among otherwise healthy college students has not been investigated. Our findings demonstrate a similar trend as seen in a 2014 study conducted among patients in clinical care in London, England [5] in that multiple biotypes and strains were observed.

Our results are different from the trend found among student health care workers in France in 2004 [7]. In 2004, the authors considered an overall estimated prevalence of MRSA carriage of $2.5 \%$ to be high when compared with the rate recorded for France. Our MRSA carriage rates are as high as $25 \%$ among those working in clinics - ten times the rate. Regarding the student population, the French researchers found no statistical association between nasal carriage of MRSA and any of the items recorded through their questionnaire. In addition, they found no significant statistical difference in the prevalence of either methicillin-susceptible $S$. aureus or MRSA between students and control subjects. Their results indicated that the two populations with higher prevalence of MRSA nasal carriage were practitioners and fellows [7].

\section{Conclusion}

We now hypothesize that many individuals carry different isolates of MRSA simultaneously and probably acquired 


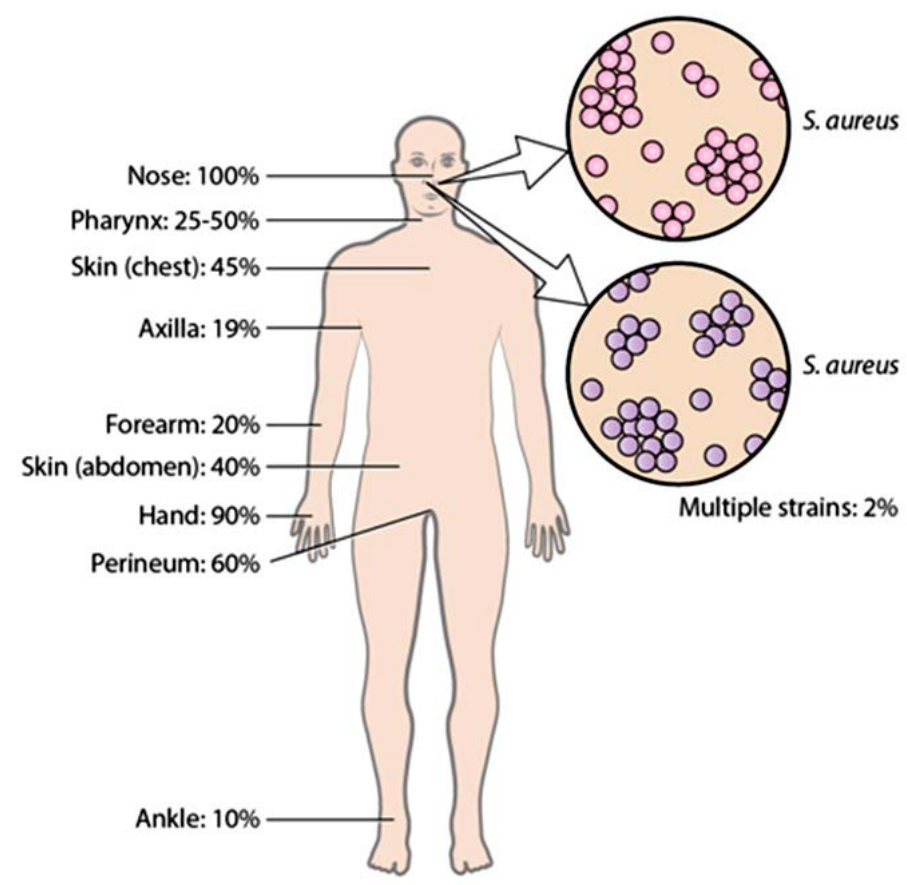

Figure 3. Published S. aureus carriage rates per body site in chronic carrier adults. There is a high carriage rate in the anterior nares among $S$. aureus carriers. The mentioned rates are approximations using data from the literature cited in the text.

the isolates from contact with individuals or patients from different environments throughout their lifetime. It could be that up to $10 \%$ people carrying MRSA have multiple origins given that $10 \%$ of $S$. aureus carriers have more than one genotype or phage type in their nose [5] [6]. Nursing students showed the highest incidence of MRSA carriage probably due to their clinical exposure, especially in nursing homes. We believe the solution to reducing infections is increasing awareness of students of high risks, hand washing, and regular testing for staphylococcal and MRSA colonization. For those students who were found MRSA positive and began using a chlorhexidine body wash and applied an appropriate antibiotic treatment (per physician prescription), they were found to MRSA-free within weeks.

Data from this study support the hypothesis that the major predictor of MRSAcarriage is clinical exposure and in particular working at nursing homes. Minor predictors of MRSA carriage are nose abnormality and lack of hygiene (nose picking, nose rings without asepsis, and minimal handwashing). In addition, a person may carry more than one MRSA strain as noted by varied antibiotic sensitivity. Future studies are needed to determine the number of multiple strains (or biotypes) that colonize students to determine the risk of infection for students that spend extended time in a clinical setting.

\section{Acknowledgements}

Financial assistance of this project was graciously donated by Liberty University's Department of Biology and Chemistry. Special thanks to Dr. David DeWitt, Yingguang Liu, Dr. Randy Hubbard, Dr. Michael Price, Abigail Lentz, the microbiology faculty, and students at Liberty University. In addition, we thank Rick Simonson for illustrating Figure 3.

\section{References}

[1] Center for Disease Control and Prevention (2013) FY15 Detect and Protect against Antibiotic Resistance Budget Initiative. http://www.cdc.gov/drugresistance/threat-report-2013/pdf/FY15-DPAR-budget-init.pdf

[2] Center for Disease Control and Prevention (2007) CDC Estimates 94,000 Invasive Drug-Resistant Staph Infections Occurred in the U.S. in 2005. http://www.cdc.gov/media/pressrel/2007/r071016.htm 
[3] CDC (2014) Methicillin-Resistant Staphylococcus aureus (MRSA) Infections. Center for Disease Control and Prevention. http://www.cdc.gov/mrsa/

[4] Kildow B, Conradie J, Robson R. (2012) Nostrils of Healthy Volunteers Are Independent with Regard to Staphylococcus aureus Carriage. Journal of Clinical Microbiology, 50, 3744-3746. http://dx.doi.org/10.1128/JCM.01488-12

[5] Votintseva, A.A., Miller, R.R., Fung, R., Knox, K., Godwin, H., Peto, T.E.A., et al. (2014) Multiple-Strain Colonization in Nasal Carriers of Staphylococcus aureus. Journal of Clinical Microbiology, 52, 1192-1200. http://dx.doi.org/10.1128/JCM.03254-13

[6] Wertheim, H., Melles, D., Vos, M., Van Leeuwen, W., Van, B., Verbrugh, H., et al. (2005) The Role of Nasal Carriage in Staphylococcus aureus Infections. The Lancet Infectious Diseases, 5, 751-762. http://dx.doi.org/10.1016/S1473-3099(05)70295-4

[7] Berthelot, P., Grattard, F., Fascia, P., et al. (2004) Is Nasal Carriage of Methicillin-Resistant Staphylococcus aureus More Prevalent among Student Healthcare Workers? Infection Control and Hospital Epidemiology, 25, 364-365. http://dx.doi.org/10.1086/503498

[8] Courvalin, P., LeClercq, R. and Rice, L.B. (Eds.) (2011) Antibiogram. ASM Press, Washington DC.

[9] Yan, M., Pamp, S., Fukuyama, J., Hwang, P., Cho, D., Holmes, S., et al. (2013) Nasal Microenvironments and Interspecific Interactions Influence Nasal Microbiota Complexity and S. aureus Carriage. Cell Host \& Microbe, 14, 631-640. http://dx.doi.org/10.1016/j.chom.2013.11.005

[10] Highlander, S., Hultén, K., Quin, X., Jiang, H., Yerrapragada, S., Mason, E., et al. (2007) Subtle Genetic Changes Enhance Virulence of Methicillin-Resistant and Sensitive Staphylococcus aureus. BMC Microbiology, 7, 1-14. http://www.biomedcentral/1471-2180/7/99.

[11] Hudson, L., Reynolds, C., Spratt, B., Enright, M., Quan, V., Kim, D., et al. (2013) Diversity of Methicillin-Resistant Staphylococcus aureus Strains Isolated from Residents of 26 Nursing Homes in Orange County, California. Journal of Clinical Microbiology, 51, 3788-3795. http://dx.doi.org/10.1128/JCM.01708-13

[12] Lee, B., Bartsch, S., Wong, K., Singh, A., Avery, T., Kim, D., et al. (2013) The Importance of Nursing Homes in the Spread of Methicillin-Resistant Staphylococcus (MRSA) among Hospitals. Medical Care, 5, 205-215. http://dx.doi.org/10.1097/MLR.0b013e3182836dc2 
Scientific Research Publishing (SCIRP) is one of the largest Open Access journal publishers. It is currently publishing more than 200 open access, online, peer-reviewed journals covering a wide range of academic disciplines. SCIRP serves the worldwide academic communities and contributes to the progress and application of science with its publication.

Other selected journals from SCIRP are listed as below. Submit your manuscript to us via either submit@scirp.org or Online Submission Portal.
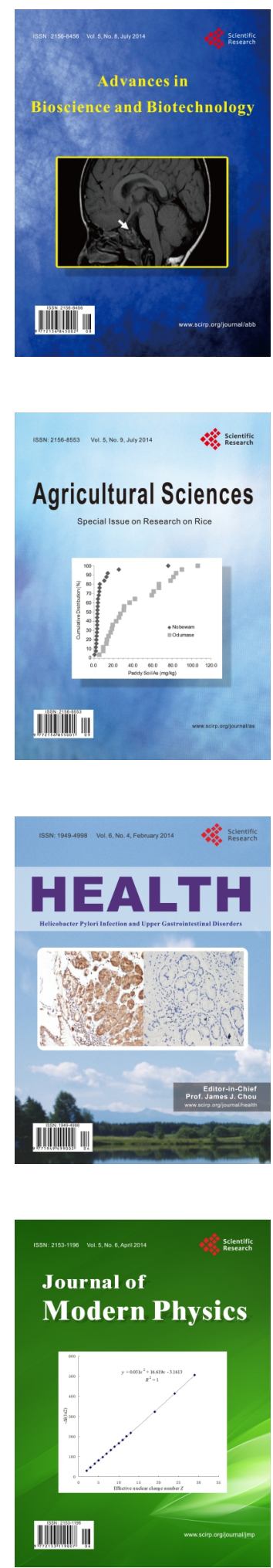
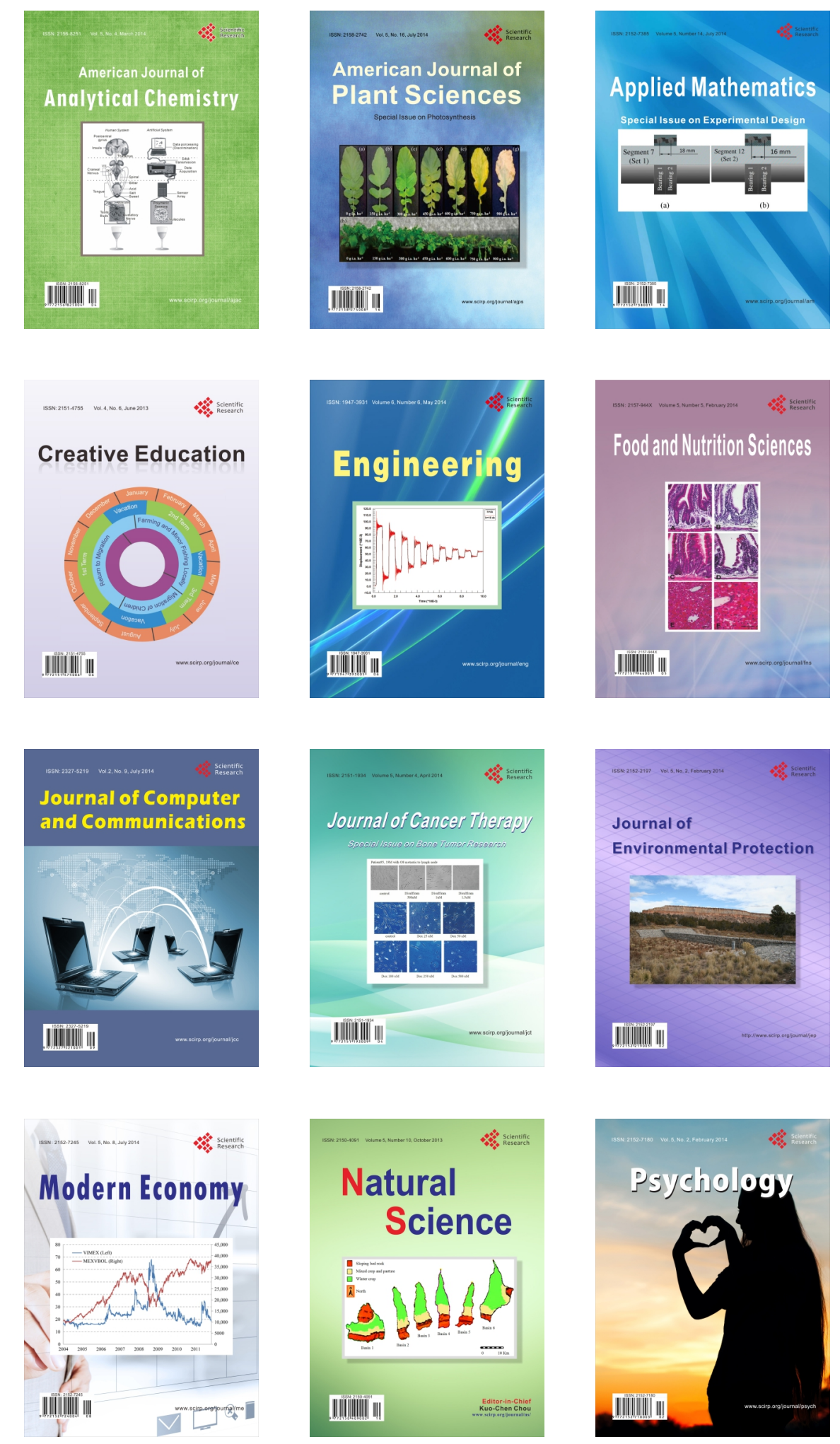